\title{
Lance-adams Syndrome - A Case Report
}

\author{
Dr M S Biradar ${ }^{1}$, Dr Alekhya Vempati ${ }^{2}$

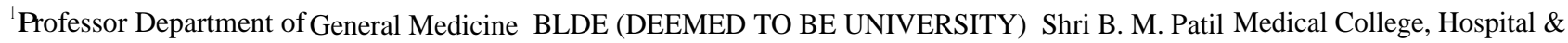 \\ Research Centre, Vijayapur, Karnataka, India \\ ${ }^{2}$ Resident Department of General Medicine, BLDE(DEEMED TO BE UNIVERSITY) Shri B. M. Patil Medical College, Hospital \& \\ Research Centre, Vijayapur, Karnataka, India
}

\section{Introduction}

Posthypoxic myoclonus (PHM) is a neurological complication characterized by uncontrolled myoclonic jerks following periods of cerebral hypoxia. The most common cause is after a successful resuscitation from a cardiac arrest where the cerebral blood flow is compromised for a period of time. Other causes include status asthamaticus, status epilepticus, coma secondary to respiratory cardiac or metabolic causes.

The acute type of PHM, which is called myoclonic status epilepticus, occurs within 12 hours in most cases after hypoxic brain damage in patients who are deeply comatose. The chronic type of PHM, which is known as Lance-Adams syndrome (LAS), is characterized by action myoclonus beginning within days to weeks after the event causing hypoxic brain injury and persists even after the patients regains consciousness. Here we present a case of Lance Adams syndrome post extubation following acute respiratory distress syndrome in a female who was ventilated and in coma for seven days.

\section{Case Report}

A 33 year old female presented with jerky movements throughout the body and difficulty in speaking since 15 days. One month back the patient had history of fever, cough with hemoptysis and difficulty in breathing which worsened over a period of 5 days. The patients was diagnosed with sepsis causing ARDS and had to be intubated for a period of 7 days during which she was in coma. The patient was extubated on the seventh day and showed improvement but she started developing myoclonic jerks in her upper and lower limbs. As the patient started ambulating, the jerky movements worsened and she started developing difficulty in speaking. The CT brain showed cerebral edema with no other lesions. On examination the patient had intact higher mental functions except for speech. She had motor dysphasia. Cranial nerves examination was normal. The motor and sensory system examinations showed no deficits. The patient had involuntary movements on attempting to walk or do any motor activity.

Routine investigations revealed no abnormalities. Repeat CT scan was normal. EEG suggested multifocal epileptogenic activity. Given the back drop of respiratory arrest and coma, post hypoxic myoclonus (Lance Adams syndrome) was diagnosed.

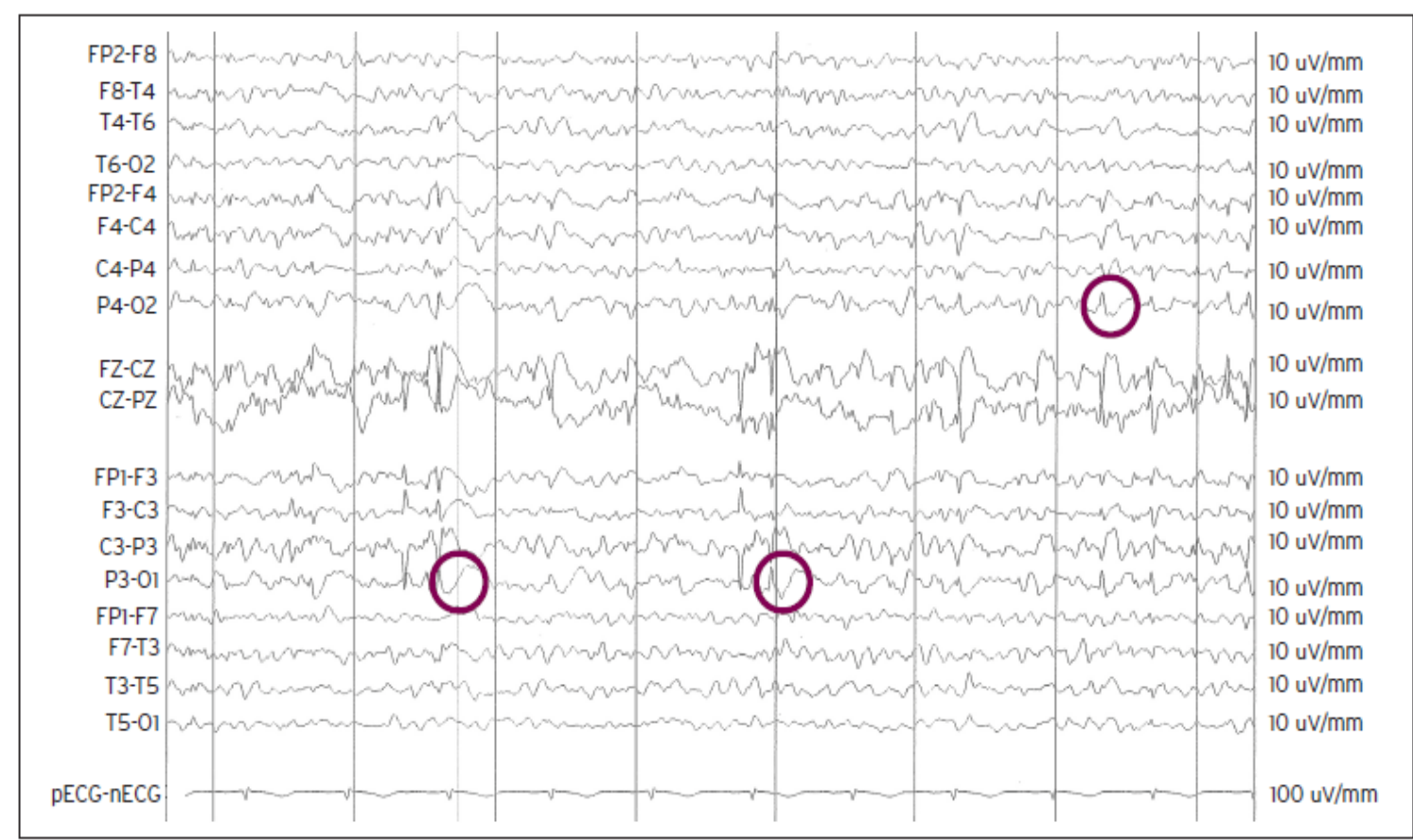

Figure: There is widespread spike and wave activity which appears to be multi-focal in origin, background rhythm appears to be reasonable with $8 \mathrm{~Hz}$ and reactive rhythm 


\section{Treatment and Follow Up}

The patient was already started on clonazepam $10 \mathrm{mg}$ and levitiracetam 500mg.We started the patient on sodium valproate $200 \mathrm{mg}$ and explained about the condition and its prognosis as the patient had been earlier diagnosed as myoclonic status epilepticus with poor prognosis. She was informed that the jerks would eventually disappear and with continued treatment would be under control. The patient was asked to follow up every 15 days. The patient improved dramatically in the follow up visits and by the end of two months the myoclonic jerks were only restricted to the upper limbs.

\section{Discussion}

LAS is characterized by myoclonus that starts days to weeks after a cardiac arrest. The myoclonus is initiated by movement, startling, and tactile stimuli and alleviated when a patient rests or sleeps. The patient may be diagnosed with LAS because he has clinical myoclonus characteristic of the patients reported by Lance and Adams. It is known that the pathophysiology of LAS is related to an abnormal finding of diverse neurochemicals, in particular, a loss of serotonin (5hydroxytryptamine or 5-HT), which 5-Hydroxytryptophan (5-HTP) is decarboxylated3, gamma-amino butyric acid (GABA). Substances like GABA affect the clinical progression of LAS by influencing the 5-HT system.

However, the exact pathophysiology of LAS is still not clear and therefore there is no guideline for drug treatment of the disease. As a result, treatment is limited and is often decided on empirically.

\section{References}

[1] Werhahn KJ, Brown P, Thompson PD, Marsden CD. The clinical features and prognosis of chronic posthypoxic myoclonus. Mov Disord 1997; 12: 216-220

[2] Lance JW, Adams RD. The syndrome of intention or action myoclonus as a sequel to hypoxic encephalopathy. Brain 1963; 86: 111-136

[3] Welsh JP, Placantonakis DG, Warsetsky SI, Marquez RG, Bernstein L, Aicher SA. The serotonin hypothesis of myoclonus from the perspective of neuronal rhythmicity. Adv Neurol 2002; 89: 307-329

[4] Matsumoto RR, Truong DD, Nguyen KD, Dang AT, Hoang TT, Vo PQ, Sandroni P. Involvement of GABA(A) receptors in myoclonus. Mov Disord 2000; 15: $47-52$ 